\title{
Hard and superconducting cubic boron phase via swarm-intelligence structural prediction driven by a machine-learning potential
}

\author{
Qiuping Yang $\odot,{ }^{1,2, *}$ Jian Lv, ${ }^{3, *}$ Qunchao Tong $\odot,{ }^{3,{ }^{*}}$ Xin Du, ${ }^{1,2}$ Yanchao Wang, ${ }^{3}$ Shoutao Zhang $\odot,{ }^{1}$ \\ Guochun Yang $\odot,{ }^{1,2, \dagger}$ Aitor Bergara $\odot,{ }^{4,5,6, \sharp}$ and Yanming $\mathrm{Ma}^{3, \S}$ \\ ${ }^{1}$ Centre for Advanced Optoelectronic Functional Materials Research and Key Laboratory for UV Light-Emitting Materials \\ and Technology of Ministry of Education, Northeast Normal University, Changchun 130024, China \\ ${ }^{2}$ State Key Laboratory of Metastable Materials Science \& Technology and Key Laboratory for Microstructural Material \\ Physics of Hebei Province, School of Science, Yanshan University, Qinhuangdao 066004, China \\ ${ }^{3}$ International Center of Computational Method and Software, State Key Laboratory of Superhard Materials, \\ College of Physics, Jilin University, Changchun 130012, China \\ ${ }^{4}$ Departamento de Física de la Materia Condensada, Universidad del País Vasco-Euskal Herriko Unibertsitatea, \\ UPV/EHU, 48080 Bilbao, Spain \\ ${ }^{5}$ Donostia International Physics Center (DIPC), 20018 Donostia, Spain \\ ${ }^{6}$ Centro de Física de Materiales CFM, Centro Mixto CSIC-UPV/EHU, 20018 Donostia, Spain
}

(Received 14 October 2020; revised 26 November 2020; accepted 16 December 2020; published 8 January 2021)

Boron is an intriguing element due to its electron deficiency and the ability to form multicenter bonds in allotropes and borides, exhibiting diversified structures, unique chemical bonds, and interesting properties. Using swarm-intelligence structural prediction driven by a machine learning potential, we identified a boron phase with a 24-atom cubic unit cell, called $c$ - $\mathrm{B}_{24}$, consisting of a $\mathrm{B}_{6}$ octahedron in addition to well-known $\mathrm{B}_{2}$ pairs and $\mathrm{B}_{12}$ icosahedra at ambient pressure. There appear unusual four-center-two-electron (4c-2e) bonds in the $\mathrm{B}_{12}$ icosahedron, originating from the peculiar bonding pattern between the $\mathrm{B}_{2}$ pair and $\mathrm{B}_{12}$ icosahedron, which is in sharp contrast with the $3 \mathrm{c}-2 \mathrm{e}$ and $2 \mathrm{c}-2 \mathrm{e}$ bonds in $\alpha-\mathrm{B}_{12}$. More interestingly, $c-\mathrm{B}_{24}$ is a metal with a superconducting critical temperature of $13.8 \mathrm{~K}$ at ambient pressure. The predicted Vickers hardness (23.1 GPa) indicates that $c-\mathrm{B}_{24}$ is a potential hard material. Notably, it also has a good shear/tensile resistance (48.9/29.3 $\mathrm{GPa}$ ). Our work not only enriches the understanding of the chemical properties of boron, but also sparks efforts on trying to synthesize this particular compound, $c-\mathrm{B}_{24}$.

DOI: 10.1103/PhysRevB.103.024505

\section{INTRODUCTION}

Allotropes, which refer to different forms of the same element, have become a fascinating area of research in condensed matter physics and chemistry [1-3]. Finding new allotropes, with unique structural units and a combination of several chemical bonds, allows a deeper understanding of the chemical behavior of the elements [4-6]. On the other hand, the preparation of allotropes is an important way to obtain functional materials with fascinating properties [7-10]. A large number of allotropes are already known, especially for the light elements, as carbon, silicon, nitrogen, and boron, but there are still interesting surprises to be discovered [11-17]. This is basically due to their complex potential energy surfaces with a plethora of local minima, which causes diversified metastable structures (i.e., allotropes) to exist [18-22]. For example, in addition to the five known nitrogen allotropes [23], an allotrope with the black phosphorus structure has been found very recently $[24,25]$.

\footnotetext{
*These authors contributed equally to this work.

†Corresponding author: yanggc468@ nenu.edu.cn

‡Corresponding author: a.bergara@ehu.eus

${ }^{\S}$ Corresponding author: mym@jlu.edu.cn
}

Boron has three valence electrons and, following the classical valence bond theory, it can only form three covalent bonds with three boron/other atoms forming allotropes or compounds. However, its ability to form multicenter bonds greatly enriches its structural diversity [26]. So far, at least 16 boron allotropes have been reported [14], with various electronic properties (e.g., semiconductivity, metallicity, semimetallicity, and superconductivity). On the other hand, the properties of boron allotropes are strongly correlated with binding patterns and the arrangement of structural units [14,21,27]. Specifically, Pnma-B ${ }_{60}$, where $\mathrm{B}_{12}$ icosahedra are linked by two-atom wide boron ribbons, is a semimetal, whereas $I 2_{1} 2_{1} 2_{1}-\mathrm{B}_{60}$, in which $\mathrm{B}_{12}$ icosahedra are interconnected by helical boron chains, is a metal [28]. Taking into account the complex potential energy surfaces and diversified binding patterns of boron $[29,30]$, which favors the formation of novel structural units or different combinations of already known structural units, it is still possible to find new boron allotropes.

Recently, theoretical prediction plays a critical and leading role in accelerating the discovery of new allotropes [22,31-34]. In this work, we employ the swarm-intelligence structural search method combined with a machine learning potential to search for new boron allotropes at ambient pressure containing up to $60 \mathrm{~B}$ atoms. In addition to 
(a)

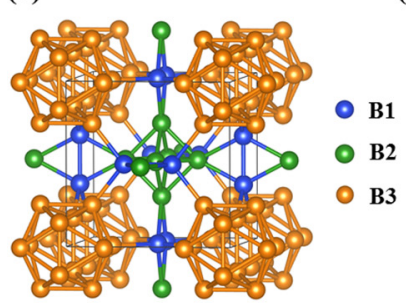

(e)

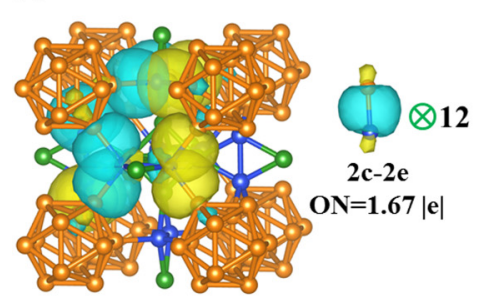

$12 \times 2 \mathrm{c}-2 \mathrm{e} \sigma$ bonds

(b)

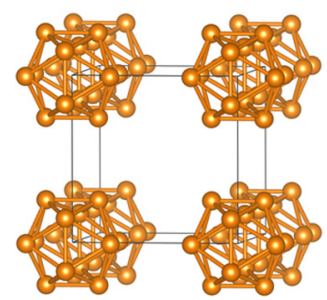

(f)

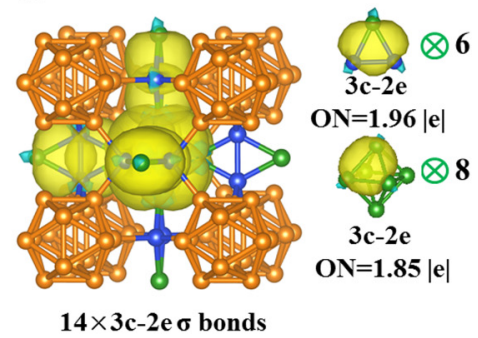

(d)

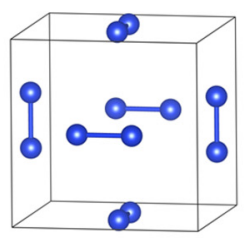

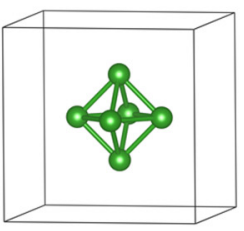

(g)

FIG. 1. (a) Crystal structure of the $c$ - $\mathrm{B}_{24}$ phase. The location of the (b) $\mathrm{B}_{12}$ icosahedra, (c) $\mathrm{B}_{2}$ pairs, and (d) $\mathrm{B}_{6}$ octahedra in the lattice. The SSAdNDP chemical bonding analysis of $c-\mathrm{B}_{24}$ along with the occupation number $(\mathrm{ON})$. In total 36 bonds are showed, including (e) 12 2c-2e $\sigma$ bonds, (f) $143 \mathrm{c}-2 \mathrm{e} \sigma$ bonds, and (g) $104 \mathrm{c}-2 \mathrm{e} \sigma+\pi$ bonds. The " + " symbol represents an overlap between the $\sigma$ and $\pi$ bonds.

replicating the four reported boron allotropes (e.g., $\alpha-\mathrm{B}_{12}$ [35], $\gamma-\mathrm{B}_{28}$ [14], $\alpha$-Ga-type $\mathrm{B}$ [36], and $\mathrm{B}_{10}$ [37]), we have found one, named $c-\mathrm{B}_{24}$, which shows a different structural unit ( $\mathrm{B}_{6}$ octahedron) situated at the center of the cubic unit cell. On the other hand, the unique arrangement between $\mathrm{B}_{12}$ icosahedron and the $B_{2}$ pair induces a $4 c-2 e$ bond in the $\mathrm{B}_{12}$ icosahedron. More interestingly, $c-\mathrm{B}_{24}$ is both hard and superconducting.

\section{COMPUTATIONAL DETAILS}

The crystal structure search is performed with the swarmintelligence CALYPSO method [38-40] driven by a modified version of the Gaussian approximation potential (GAP) [41]. Compared with the original GAP [42], the atomic-centered symmetry function [43], rather than the smooth overlap of atomic positions [44], was used as descriptors to describe neighboring environment of $\mathrm{B}$ atoms. The CALYPSO method has been successfully applied in many systems, including $\mathrm{C}_{84}$ clusters, [41] $\mathrm{C}_{10}$ [45], $\mathrm{TiO}_{2}$ [46], and some organic materials [47]. In this work, the already known four stable boron phases $\left(\alpha-\mathrm{B}_{12}, \gamma-\mathrm{B}_{28}, \alpha\right.$-Ga-type $\mathrm{B}$, and $\left.\mathrm{B}_{10}\right)$ are successfully replicated $[14,37,48-52]$, demonstrating the reliability of the method in the global structure search. More detailed information can be found in the Supplemental Material [53].

The underlying structural relaxation and electronic properties are carried out by employing the Vienna $a b$ initio simulation package (VASP) $[54,55]$. The electron-ion interaction is represented by the full electron projection augmented wave method [56], and $2 s^{2} 2 p^{1}$ are treated as valence electrons of $\mathrm{B}$. The plane wave basis with a kinetic energy cutoff of $800 \mathrm{eV}$ is used, and a Monkhorst-Pack scheme [57] with a dense $k$-point grid spacing of $2 \pi \times 0.03 \AA^{-1}$ is chosen to ensure a good convergence of the total energy. Phonon calculations are performed by using the finite displacement approach [58] in the PHONOPY code [59]. First-principles molecular dynamics simulations [60] using the canonical $N V T$ are performed with a time step of 1 fs to evaluate the thermal stability of the predicted structures. Electron-phonon coupling (EPC) calculations were performed with density functional perturbation theory using the Quantum-ESPRESSO package [61].

\section{RESULTS AND DISCUSSION}

\section{A. Structure, chemical bonding, and stability}

After an extensive structural search at ambient pressure, we found a cubic structure with the space group $P m \overline{3}$, containing 24 atoms in the unit cell, named $c-\mathrm{B}_{24}$ [Fig. 1(a)]. Within this structure, three inequivalent $\mathrm{B}$ atoms occupy the crystallographic $6 \mathrm{~g}(0.3528,0.5000,0.0000)$, 6h $(0.7204,0.5000$, $0.5000)$, and $12 \mathrm{j}(0.0000,0.8414,0.2540)$ sites, which form three kinds of structural units, i.e., $\mathrm{B}_{6}$ octahedron, $\mathrm{B}_{2}$ pair, and $\mathrm{B}_{12}$ icosahedron, Figs. 1(b)-1(d), located at the body center, face center, and vertex of the cubic lattice, respectively. The $\mathrm{B}$ atoms in $\mathrm{B}_{2}$ units are connected with the vertex $\mathrm{B}$ atoms in the $\mathrm{B}_{12}$ icosahedra and the $\mathrm{B}_{6}$ octahedron to form a dense three-dimensional packing structure. Notably, $\mathrm{B}_{6}$ octahedron appears in the reported boron allotropes, but it has also been observed in metal borides at ambient (e.g., $\mathrm{BeB}_{6}, \mathrm{MgB}_{6}$, $\mathrm{CaB}_{6}, \mathrm{LaB}_{6}$, and $\mathrm{YB}_{6}$ ) [62-64] and high pressures (e.g., $\mathrm{BaB}_{6}$ and $\left.\mathrm{KB}_{6}\right)[65,66]$.

It is well-known that $\mathrm{B}$ atoms tend to form multicenter bonds, playing a key role in stabilizing allotropes and B-rich borides [26,67]. Furthermore, the presence of $\mathrm{B}_{6}$ octahedra and the unique bonds between $B_{2}$ structural units and $B_{12}$ icosahedra lead us to explore the characteristics of these chemical bonds. Here, the solid state adaptive natural density partitioning (SSAdNDP) method [68], whose efficacy has been verified in a plethora of compounds $[69,70]$, is used to study the binding patterns in $c$ - $\mathrm{B}_{24}$. The unit cell of $c-\mathrm{B}_{24}$, with 72 valence electrons, presents 12 2c-2e bonds at the junction between $B_{12}$ icosahedra and $B_{2}$ pairs [Fig. 1(e)], eight $3 c-2 e$ bonds in $\mathrm{B}_{6}$ octahedra, and six $3 c-2 e$ bonds at the intersection between $\mathrm{B}_{6}$ octahedra and $\mathrm{B}_{2}$ pairs [Fig. 1(f)]. More 


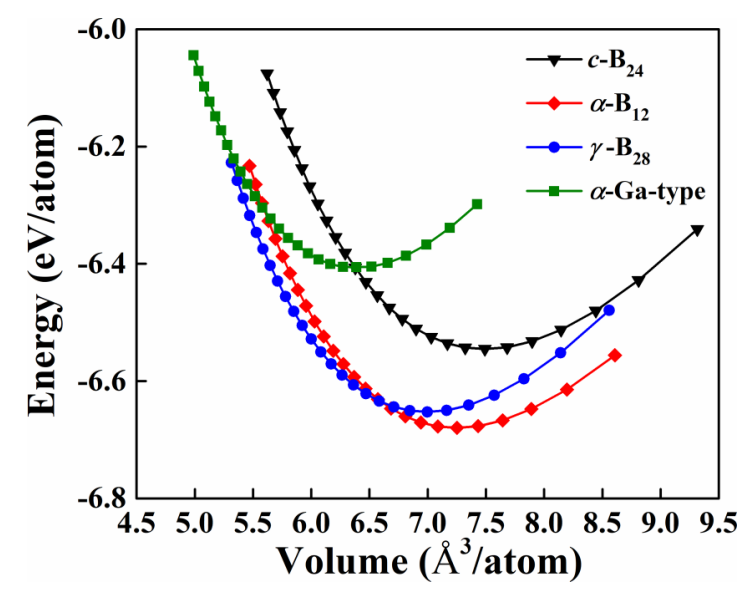

FIG. 2. Total energy of $c-\mathrm{B}_{24}$ as a function of the volume per atom in comparison with those of $\alpha-\mathrm{B}_{12}, \gamma-\mathrm{B}_{28}$, and $\alpha$-Ga-type boron.

interestingly still, $104 \mathrm{c}-2 \mathrm{e}$ bonds appear in the $\mathrm{B}_{12}$ icosahedral unit [Fig. $1(\mathrm{~g})]$, which is in sharp contrast with the $\mathrm{B}_{12}$ icosahedron in $\alpha$ - $\mathrm{B}_{12}$ (i.e., with strong $2 \mathrm{c}-2 \mathrm{e}$ and weak $3 \mathrm{c}-$ 2e bonds) $[14,71]$. This could be attributed to their different atomic arrangements. Specifically, in $c-\mathrm{B}_{24}$ every boron atom in the icosahedral unit $\mathrm{B}_{12}$ is bonded to $\mathrm{B}$ atoms in $\mathrm{B}_{2}$ units, forming $12 \mathrm{~B}-\mathrm{B}$ bonds (Fig. S2). In $\alpha-\mathrm{B}_{12}$, the icosahedral units connect each other with six pairs of $\mathrm{B}-\mathrm{B}$ bonds. The $4 \mathrm{c}-2 \mathrm{e}$ bonds have been observed in two-dimensional (2D) materials, such as the $\alpha$-boron sheet [69], and have also been reported in boron triangle units of bulk $\alpha^{\prime}$ boron [72], whose atomic arrangement is completely different from the $\mathrm{B}_{12}$ icosahedron. The unique bonding combination might induce different electronic properties. In addition, the average $\mathrm{B}-\mathrm{B}$ bond length in $c-\mathrm{B}_{24}$ is $1.77 \AA$, which is equal and slightly shorter than $1.77 \AA$ in $\alpha-\mathrm{B}_{12}$ and $1.82 \AA$ in $\gamma-\mathrm{B}_{28}$. The average Mulliken overlap population of $c-\mathrm{B}_{24}(0.63)$ is slightly larger than that of $\alpha-\mathrm{B}_{12}(0.56)$ and $\gamma-\mathrm{B}_{28}(0.55)$, indicating that $\mathrm{B}-\mathrm{B}$ bonds in $c-\mathrm{B}_{24}$ are stronger.

The thermodynamic stability of the predicted structure can be used to evaluate the feasibility of the experimental synthesis. Figure 2 shows the calculated total energy versus volume of $c-\mathrm{B}_{24}$, compared to the three already reported boron allotropes (e.g., $\alpha$ - $\mathrm{B}_{12}$ [35], $\gamma-\mathrm{B}_{28}$ [14], and $\alpha$-Ga-type B [36]). At ambient conditions, it can be clearly seen that $\alpha-B_{12}$ has the lowest value of the total energy per atom, and $\alpha$-Gatype boron shows the maximum one, which is consistent with previous reports [14] and supports the reliability of our calculations. At ambient pressure, $c-\mathrm{B}_{24}$ is $0.139 \mathrm{eV} /$ atom lower in energy than $\alpha$-Ga-type boron, but 0.134 and $0.107 \mathrm{eV} /$ atom higher in energy than $\alpha-\mathrm{B}_{12}$ and $\gamma-\mathrm{B}_{28}$, respectively. Several metastable phases have been synthesized with much higher energy than the most stable phase, such as $M$-carbon ( 0.283 eV/atom higher than graphite) [12], the nitrogen allotrope with black phosphorus structure $(1.12 \mathrm{eV} /$ atom higher than $\alpha$-N) [24], and the Si-III structure (0.16 eV/atom higher than $\mathrm{Si}-\mathrm{I})$ [73]. These results imply that $c$ - $\mathrm{B}_{24}$ could be synthesized in certain conditions and exist as a metastable phase.

Phonon dispersions of $c-\mathrm{B}_{24}$ without any imaginary frequency in the whole Brillouin zone demonstrate that it is dynamically stable at both ambient (Fig. S3) and high (a)

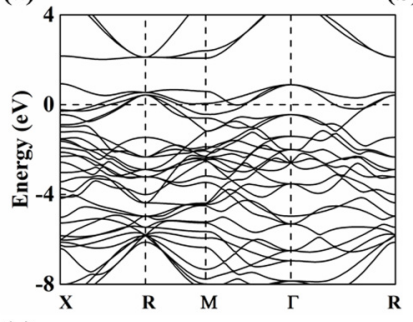

(c)

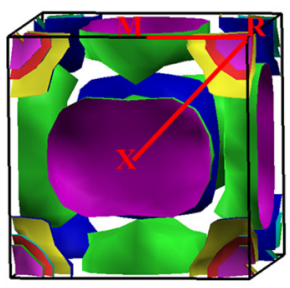

(e)

(d)

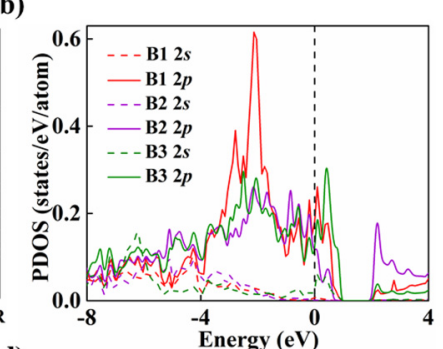

(d)
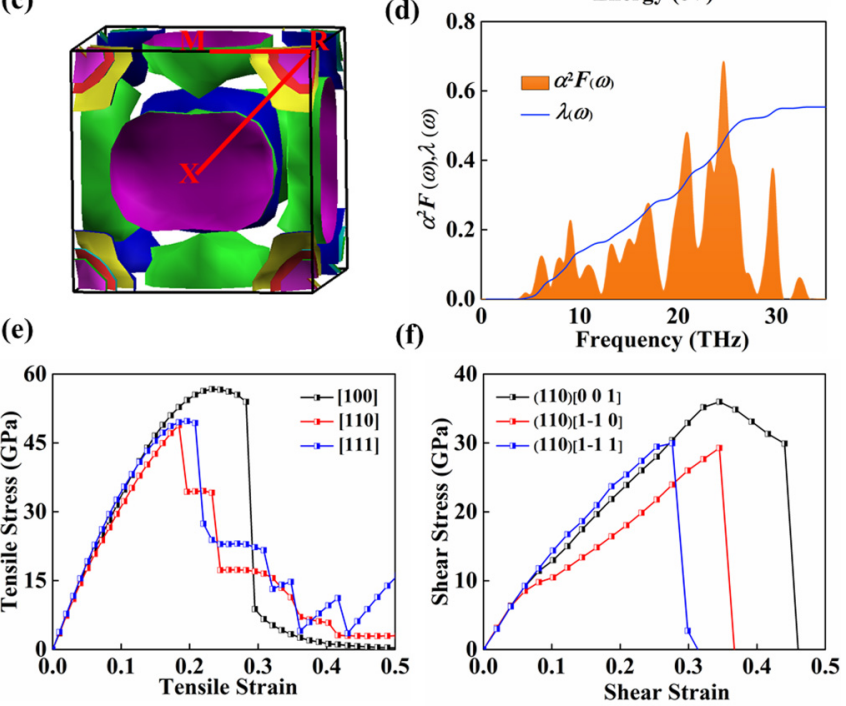

FIG. 3. (a) The electronic band structure of $c-\mathrm{B}_{24}$ at $1 \mathrm{~atm}$. (b) Projected density of states (PDOS) of $c-\mathrm{B}_{24}$ with different occupying sites at $1 \mathrm{~atm}$. (c) The Fermi surface for $c-\mathrm{B}_{24}$ at $1 \mathrm{~atm}$. (Fig. S5 shows the Fermi surface associated with each band crossing the Fermi level.) (d) The Eliashberg spectral function $\alpha^{2} F(\omega)$ and integrated electron-phonon coupling parameters $\lambda(\omega)$ of $c-\mathrm{B}_{24}$ at $1 \mathrm{~atm}$. (e) The calculated stress-strain relations of phase $c-\mathrm{B}_{24}$ in the three stress tension directions. (f) The calculated stress-shear for $c$ - $\mathrm{B}_{24}$ in the three principal symmetry crystallographic directions.

pressures (i.e., 25 and $50 \mathrm{GPa}$, Fig. S3). To examine the thermal stability, ab initio molecular dynamics simulations are also performed with the canonical $(N V T)$ ensemble at $1000 \mathrm{~K}$, using the Nosé thermostat [60] with a step of $1 \mathrm{fs}$. We model the system using a $2 \times 2 \times 2$ supercell that has $192 \mathrm{~B}$ atoms. Energy fluctuations at $1000 \mathrm{~K}$ are shown in Fig. S4, and the initial and final structures are also presented. Interestingly, the framework of $c-\mathrm{B}_{24}$ maintains its original configuration at $1000 \mathrm{~K}$, exhibiting an outstanding thermal stability. Furthermore, $c-\mathrm{B}_{24}$ satisfies the criteria for mechanical stability, as will be discussed later.

\section{B. Electronic properties and superconducting}

The appearance of different $B_{6}$ octahedra and unique $4 c-2 e$ bonds in the $\mathrm{B}_{12}$ icosahedra stimulate us to explore its electronic properties. The calculated electronic band structure and the corresponding projected density of states (PDOS) at the Perdew-Burke-Ernzerhof (PBE) level are shown in Figs. 3(a) and 3(b). Unexpectedly, $c-\mathrm{B}_{24}$ is a metal with several bands crossing the Fermi level, which is in sharp contrast with the nonmetallic $\alpha-\mathrm{B}_{12}$ [14] and $\gamma-\mathrm{B}_{28}$ [14] but similar to the 
$\alpha$-Ga-type boron [36] at ambient pressure. PDOS analysis shows that its metallicity comes mainly from the contribution of $\mathrm{B} 2 p$. On the other hand, there is a strong overlap between $\mathrm{B} 2 p$ in $\mathrm{B}_{2}$ units and $\mathrm{B} 2 p$ in $\mathrm{B}_{6}$ octahedra and $\mathrm{B}_{12}$ icosahedra, indicating the formation of strong chemical bonds, in line with the previous chemical bond analysis. The contribution of $\mathrm{B} 2 s$ in $\mathrm{B}_{12}$ icosahedra to metallicity is much higher than that of the $B_{2}$ units and $B_{6}$ octahedra, which could originate from the unique $4 c-2 e$ bonds in the $B_{12}$ icosahedra. In addition, a well-defined Fermi surface nesting appears along $X \rightarrow R$ and $\mathrm{R} \rightarrow \mathrm{M}$ [Fig. 3(c)], with highly dispersive bands in the two directions. On the contrary, flatter bands associated with more localized electronic states appear along $\mathrm{X} \rightarrow \mathrm{R}$, which induces a high electronic density of states near the Fermi level.

Motivated by the strong PDOS peak at the Fermi level and the presence of steep bands along the $\Gamma \rightarrow \mathrm{M}$ direction and flat bands along the $\mathrm{X} \rightarrow \mathrm{R}$ direction, we have explored the superconductivity of $c-\mathrm{B}_{24}$. The superconducting transition temperature $\left(T_{\mathrm{c}}\right)$ was estimated by using the Allen and Dynes modified McMillan equation with a typical choice of $\mu^{*}=0.1$ [74-77]. The calculated $T_{\mathrm{c}}$ of $c-\mathrm{B}_{24}$ is $13.8 \mathrm{~K}$ at ambient pressure, becoming the first superconductor among known bulk boron allotropes at $0 \mathrm{GPa}$, and is much higher than $6 \mathrm{~K}$ of $\beta$-B at $175 \mathrm{GPa}$ [78] and comparable to 2D B polymorphs (e.g., $18.7 \mathrm{~K}$ for $\beta_{12}$, [79] $24.7 \mathrm{~K}$ for $\chi^{3}$ [79], and $17.9 \mathrm{~K}$ for $2 \mathrm{D}$ boron layer [66]).

The integrated electron-phonon coupling parameter $\lambda(\omega)$ and Eliashberg spectral function $\alpha^{2} F(\omega)$ are shown in Fig. 3(d). The calculated $\lambda$ is 0.55 , which is higher than 0.38 , 0.39 , and 0.39 at $160,215,273 \mathrm{GPa}$ of $\alpha$-Ga-type boron [80], 0.39 for the boron-doped diamond with $2.78 \%$ boron content [81] and comparable to 0.61 in $\mathrm{MgB}_{2}$ [82]. The contribution of low-, mid-, and high-frequency modes to the EPC are 29.5\% (below $12 \mathrm{THz}), 22 \%(12 \sim 19 \mathrm{THz})$, and $48.4 \%$ (above $19 \mathrm{THz}$ ), respectively. Therefore, its superconducting mechanism is different from the low-frequency P-derived vibrations of the $\mathrm{Li}_{6} \mathrm{P}$ electride [83], high-frequency $\mathrm{H}$-derived vibrations of high- $T_{\mathrm{c}} \mathrm{H}_{3} \mathrm{~S}$ [84], and intermediate-frequency $\mathrm{H}$ derived vibrations of $\mathrm{H}_{4} \mathrm{Te}$ [85]. We also explored its pressure dependence $T_{\mathrm{c}}$. As shown in Fig. S6, the $T_{\mathrm{c}}$ of $c-\mathrm{B}_{24}$ increases with pressure (e.g., $14.6 \mathrm{~K}$ at 25 and $17.1 \mathrm{~K}$ at $50 \mathrm{GPa}$ ), which mainly originates from the pressure-induced phonon softening along $X \rightarrow R$ in the Brillouin zone (Fig. S3), leading to an enhancement of $\lambda$.

\section{Mechanical property and hardness}

The calculated bulk modulus $(B)$, shear modulus $(G)$, Young's modulus $(E)$, and Poisson's ratio (v) of $c$ - $\mathrm{B}_{24}$ are given in Table S3. Overall, $B$ is a measure of the tensile strength of the material. The higher $B$ is, the more incompressible it is. The bulk modulus $(B)$ of $c-\mathrm{B}_{24}$ is 209.38 $\mathrm{GPa}$, which is comparable to other known hard materials [e.g., $\mathrm{Al}_{2} \mathrm{O}_{3}(254 \mathrm{GPa})$ and $\left.\mathrm{AlN}(205 \mathrm{GPa})\right][86,87]$.The ratio between the bulk modulus and the shear modulus $(B / G)$ is used to measure the brittle or ductile behavior of materials [88]. The calculated $B / G$ of $c-\mathrm{B}_{24}$ is 1.373 , which is much lower than the criterion value (1.75) for considering a material to be ductile. In addition, Poisson's ratio ( $v$ ) provides a useful information about the characteristics of the bonding forces. The resulting Poisson's ratio of $c-\mathrm{B}_{24}$ is 0.207 , which shows a large lateral expansion when it is compressed [89]. Finally, the calculated Vickers hardness value of $c-\mathrm{B}_{24}$ is $23.1 \mathrm{GPa}$, which is less than that of the recent superhard boron, orthorhombic $\gamma-\mathrm{B}_{28}$ (50 GPa) [90]. It is comparable to some typical hard materials [87] [e.g., WC (30 GPa), TiN (23 GPa), ZrC (25.8 GPa), and TiC (24.7 GPa)]. Moreover, stress-strain relations can reflect the ability of a material to resist shear and tension. The calculated tensile stress-strain relations of $c-\mathrm{B}_{24}$ in the three principal symmetry crystallographic directions ([001], [110], and [111]) are presented in Fig. 3(e). When the critical tensile stain $(\varepsilon)$ is above 0.18 , the $\mathrm{B}-\mathrm{B}$ bonds between $\mathrm{B}_{2}$ units and $\mathrm{B}_{12}$ icosahedra are broken (Fig. S7) along the [110] direction, corresponding to a peak stress of $48.9 \mathrm{GPa}$. Tensile stress along the [001] direction has the lowest peak value in the (110) plane [Fig. 3(f)] and the shear direction [1-10] shows a peak value of $29.3 \mathrm{GPa}$.

\section{CONCLUSIONS}

In search of possible boron allotropes, we explored configurations that have up to 60 atoms with the help of swarm-intelligence structural search in combination with the state-of-art machine learning potential at ambient pressure. A hitherto unknown pristine 3D crystalline boron, $c$ - $\mathrm{B}_{24}$, is identified, that exhibits high dynamical, mechanical, and thermal stability, as well as an alternative chemical bonding pattern (i.e., $4 \mathrm{c}-2 \mathrm{e}$ bond) in the $\mathrm{B}_{12}$ icosahedron. Interestingly, $c$ - $\mathrm{B}_{24}$ shows superconductivity with a $T_{\mathrm{c}}$ value of $13.8 \mathrm{~K}$ at ambient pressure; additionally, considering its predicted Vickers hardness of $23.1 \mathrm{GPa}$, as well as its good tensile and shear strengths of 48.9 and $29.3 \mathrm{GPa}$, respectively. Our work should help to expand the members of the boron family, and promote further investigations on different boron allotropes with desirable electronic and mechanical properties.

\section{ACKNOWLEDGMENTS}

The authors acknowledge the funding support from the Natural Science Foundation of China under Grants No. 21873017, No. 21573037, No. 11704062, and No. 51732003, the Postdoctoral Science Foundation of China under Grant No. 2013M541283, the Natural Science Foundation of Jilin Province (20190201231JC), the "111" Project (No. B13013). The work was carried out at National Supercomputer Center in Tianjin, and the calculations were performed on TianHe-1 (A). A.B. acknowledges financial support from the Spanish Ministry of Science and Innovation (PID2019-105488GBI00) and from Jilin Province Out-standing Young Talents project (Grant No. 20190103040JH).
[1] W. B. Jensen, J. Chem. Educ. 83, 838 (2006).

[2] A. Hirsch, Nat. Mater. 9, 868 (2010).
[3] V. Georgakilas, J. A. Perman, J. Tucek, and R. Zboril, Chem. Rev. 115, 4744 (2015). 
[4] S. Zhang, J. Zhou, Q. Wang, X. Chen, Y. Kawazoe, and P. Jena, Proc. Natl. Acad. Sci. USA 112, 2372 (2015).

[5] Q. Wang, B. Xu, J. Sun, H. Liu, Z. Zhao, D. Yu, C. Fan, and J. He, J. Am. Chem. Soc. 136, 9826 (2014).

[6] K. Umemoto, R. M. Wentzcovitch, S. Saito, and T. Miyake, Phys. Rev. Lett. 104, 125504 (2010).

[7] F. Diederich and Y. Rubin, Angew Chem. Int. Ed. English 31, 1101 (1992).

[8] M. I. Eremets, A. G. Gavriliuk, I. A. Trojan, D. A. Dzivenko, and R. Boehler, Nat. Mater. 3, 558 (2004).

[9] D. Tomasino, M. Kim, J. Smith, and C.-S. Yoo, Phys. Rev. Lett. 113, 205502 (2014).

[10] D. Laniel, G. Geneste, G. Weck, M. Mezouar, and P. Loubeyre, Phys. Rev. Lett. 122, 066001 (2019).

[11] T. Irifune, A. Kurio, S. Sakamoto, T. Inoue, and H. Sumiya, Nature (London) 421, 599 (2003).

[12] Q. Li, Y. Ma, A. R. Oganov, H. Wang, H. Wang, Y. Xu, T. Cui, H.-K. Mao, and G. Zou, Phys. Rev. Lett. 102, 175506 (2009).

[13] Z. Zhao, B. Xu, X.-F. Zhou, L.-M. Wang, B. Wen, J. He, Z. Liu, H.-T. Wang, and Y. Tian, Phys. Rev. Lett. 107, 215502 (2011).

[14] A. R. Oganov, J. Chen, C. Gatti, Y. Ma, Y. Ma, C. W. Glass, Z. Liu, T. Yu, O. O. Kurakevych, and V. L. Solozhenko, Nature (London) 457, 863 (2009).

[15] W. D. Mattson, D. Sanchez-Portal, S. Chiesa, and R. M. Martin, Phys. Rev. Lett. 93, 125501 (2004).

[16] B. Hirshberg, R. B. Gerber, and A. I. Krylov, Nat. Chem. 6, 52 (2014).

[17] X. Wu, X. Shi, M. Yao, S. Liu, X. Yang, L. Zhu, T. Cui, and B. Liu, Carbon 123, 311 (2017).

[18] B. D. Malone and M. L. Cohen, Phys. Rev. B 85, 024116 (2012).

[19] M. Xing, B. Li, Z. Yu, and Q. Chen, Materials 9, 484 (2016).

[20] D. Fan, S. Lu, A. A. Golov, A. A. Kabanov, and X. Hu, J. Chem. Phys. 149, 114702 (2018).

[21] C. Fan, J. Li, and L. Wang, Sci. Rep. 4, 6786 (2014).

[22] D. Li, F. Tian, B. Chu, D. Duan, S. Wei, Y. Lv, H. Zhang, L. Wang, N. Lu, B. Liu, and T. Cui, J. Mater. Chem. A 3, 10448 (2015).

[23] L. Lei, Q.-Q. Tang, F. Zhang, S. Liu, B.-B. Wu, and C.-Y. Zhou, Chin. Phys. Lett. 37, 068101 (2020).

[24] C. Ji, C. Ji, A. A. Adeleke, L. Yang, B. Wan, H. Gou, Y. Yao, B. Li, Y. Meng, J. S. Smith, V. B. Prakapenka, W. Liu, G. Shen, W. L. Mao, and H. K. Mao, Sci. Adv. 6, eaba9206 (2020).

[25] D. Laniel, B. Winkler, T. Fedotenko, A. Pakhomova, S. Chariton, V. Milman, V. Prakapenka, L. Dubrovinsky, and N. Dubrovinskaia, Phys. Rev. Lett. 124, 216001 (2020).

[26] H. Zhang, Y. Li, J. Hou, K. Tu, and Z. Chen, J. Am. Chem. Soc. 138, 5644 (2016).

[27] K. Shirai, Jpn. J. Appl. Phys. 56, 05FA06 (2017).

[28] X.-L. He, X. Shao, T. Chen, Y.-K. Tai, X.-J. Weng, Q. Chen, X. Dong, G. Gao, J. Sun, X.-F. Zhou, Y. Tian, and H.-T. Wang, Phys. Rev. B 99, 184111 (2019).

[29] V. L. Deringer, C. J. Pickard, and G. Csányi, Phys. Rev. Lett. 120, 156001 (2018).

[30] E. V. Podryabinkin, E. V. Tikhonov, A. V. Shapeev, and A. R. Oganov, Phys. Rev. B 99, 064114 (2019).

[31] J. Zhang, R. Wang, X. Zhu, A. Pan, C. Han, X. Li, D. Zhao, C. Ma, W. Wang, H. Su, and C. Niu, Nat. Commun. 8, 683 (2017).

[32] S. Liu, L. Zhao, M. Yao, M. Miao, and B. Liu, Adv. Sci. 7, 1902320 (2020).
[33] J. Kotakoski and K. Albe, Phys. Rev. B 77, 144109 (2008).

[34] Q. Fan, H. Wang, Y. Song, W. Zhang, and S. Yun, Comput. Mater. Sci. 178, 109634 (2020).

[35] A. R. Oganov and V. L. Solozhenko, J. Superhard. Mater. 31, 285 (2009).

[36] U. Häussermann, S. I. Simak, R. Ahuja, and B. Johansson, Phys. Rev. Lett. 90, 065701 (2003).

[37] D. Li, K. Bao, F. Tian, X. Jin, D. Duan, Z. He, B. Liu, and T. Cui, RSC Adv. 4, 203 (2014).

[38] Y. Wang, J. Lv, L. Zhu, and Y. Ma, Phys. Rev. B 82, 094116 (2010).

[39] Y. Wang, J. Lv, L. Zhu, and Y. Ma, Comput. Phys. Commun. 183, 2063 (2012).

[40] B. Gao, P. Gao, S. Lu, J. Lv, Y. Wang, and Y. Ma, Sci. Bull. 64, 301 (2019).

[41] Q. Tong, L. Xue, J. Lv, Y. Wang, and Y. Ma, Faraday Discuss 211, 31 (2018).

[42] A. P. Bartók, M. C. Payne, R. Kondor, and G. Csányi, Phys. Rev. Lett. 104, 136403 (2010).

[43] J. Behler, J. Chem. Phys. 134, 074106 (2011).

[44] A. P. Bartók, R. Kondor, and G. Csányi, Phys. Rev. B 87, 184115 (2013).

[45] Q. Wei, W. Tong, R. Yang, H. Yan, B. Wei, M. Zhang, X. Yang, and R. Zhang, Phys. Lett. A 383, 125861 (2019).

[46] N. Artrith and A. Urban, Comput. Mater. Sci. 114, 135 (2016).

[47] S. Lu, Q. Zhou, Y. Ouyang, Y. Guo, Q. Li, and J. Wang, Nat. Commun. 9, 3405 (2018).

[48] A. Masago, K. Shirai, and H. Katayama-Yoshida, Phys. Rev. B 73, 104102 (2006).

[49] B. F. Decker and J. S. Kasper, Acta Cryst. 12, 503 (1959).

[50] E. Yu Zarechnaya, L. Dubrovinsky, N. Dubrovinskaia, N. Miyajima, Y. Filinchuk, D. Chernyshov, and V. Dmitriev, Sci. Technol. Adv. Mater. 9, 044209 (2009).

[51] T. Ogitsu, E. Schwegler, and G. Galli, Chem. Rev. 113, 3425 (2013).

[52] I. Chuvashova, E. Bykova, M. Bykov, V. Prakapenka, K. Glazyrin, M. Mezouar, L. Dubrovinsky, and N. Dubrovinskaia, Phys. Rev. B 95, 180102(R) (2017).

[53] See Supplemental Material at http://link.aps.org/supplemental/ 10.1103/PhysRevB.103.024505 for computational details. Phonon dispersion curves, molecular dynamics, and the crystal structural information of $c$ - $\mathrm{B}_{24}$.

[54] G. Kresse and J. Furthmüller, Phys. Rev. B 54, 11169 (1996).

[55] G. Kresse and D. Joubert, Phys. Rev. B 59, 1758 (1999).

[56] P. E. Blöchl, Phys. Rev. B 50, 17953 (1994).

[57] H. J. Monkhorst and J. D. Pack, Phys. Rev. B 13, 5188 (1976).

[58] K. Parlinski, Z. Q. Li, and Y. Kawazoe, Phys. Rev. Lett. 78, 4063 (1997).

[59] A. Togo, F. Oba, and I. Tanaka, Phys. Rev. B 78, 134106 (2008).

[60] G. J. Martyna, M. L. Klein, and M. Tuckerman, J. Chem. Phys. 97, 2635 (1992).

[61] P. Giannozzi, S. Baroni, N. Bonini, M. Calandra, R. Car, C. Cavazzoni, D. Ceresoli, G. L. Chiarotti, M. Cococcioni, I. Dabo, A. Dal Corso, S. de Gironcoli, S. Fabris, G. Fratesi, R. Gebauer, U. Gerstmann, C. Gougoussis, A. Kokalj, M. Lazzeri, L. Martin-Samos, N. Marzari, F. Mauri, R. Mazzarello, S. Paolini, A. Pasquarello, L. Paulatto, C. Sbraccia, S. Scandolo, G. Sclauzero, A. P. Seitsonen, A. Smogunov, P. Umari, and R. M. Wentzcovitch, J. Phys.: Condens. Matter 21, 395502 (2009). 
[62] J. Etourneau, J.-P. Mercurio, and P. Hagenmuller, in Boron Refract Borides, edited by V. I. Matkovich (Springer, Berlin, 1977), pp. 115-138.

[63] I. Popov, N. Baadji, and S. Sanvito, Phys. Rev. Lett. 108, 107205 (2012).

[64] J. Etourneau and P. Hagenmuller, Philos. Mag. Part B 52, 589 (1985).

[65] X. Li, X. Huang, D. Duan, G. Wu, M. Liu, Q. Zhuang, S. Wei, Y. Huang, F. Li, Q. Zhou, B. Liu, and T. Cui, RSC Adv. 6, 18077 (2016).

[66] T. Chen, Q. Gu, Q. Chen, X. Wang, C. J. Pickard, R. J. Needs, D. Xing, and J. Sun, Phys. Rev. B 101, 054518 (2020).

[67] X. Sun, X. Liu, J. Yin, J. Yu, Y. Li, Y. Hang, X. Zhou, M. Yu, J. Li, G. Tai, and W. Guo, Adv. Funct. Mater. 27, 1603300 (2017).

[68] D. Y. Zubarev and A. I. Boldyrev, Phys. Chem. Chem. Phys. 10, 5207 (2008).

[69] T. R. Galeev, B. D. Dunnington, J. R. Schmidt, and A. I. Boldyrev, Phys. Chem. Chem. Phys. 15, 5022 (2013).

[70] H. Zhang, Y. Li, J. Hou, A. Du, and Z. Chen, Nano Lett. 16, 6124 (2016).

[71] H. Wang and Q. An, J. Phys. Chem. C 123, 12505 (2019).

[72] Y. Gao, Y. Xie, Y. Chen, J. Gu, and Z. Chen, Phys. Chem. Chem. Phys. 20, 23500 (2018).

[73] Z. Zhao, F. Tian, X. Dong, Q. Li, Q. Wang, H. Wang, X. Zhong, B. Xu, D. Yu, J. He, H.-T. Wang, Y. Ma, and Y. Tian, J. Am. Chem. Soc. 134, 12362 (2012).

[74] W. L. McMillan, Phys. Rev. 167, 331 (1968).

[75] P. B. Allen and R. C. Dynes, Phys. Rev. B 12, 905 (1975).

[76] J. P. Carbotte, Rev. Mod. Phys. 62, 1027 (1990).
[77] L. N. Oliveira, E. K. U. Gross, and W. Kohn, Phys. Rev. Lett. 60, 2430 (1988).

[78] M. I. Eremets, V. V Struzhkin, H. Mao, and R. J. Hemley, Science 293, 272 (2001).

[79] M. Gao, Q.-Z. Li, X.-W. Yan, and J. Wang, Phys. Rev. B 95, 024505 (2017).

[80] Y. Ma, J. S. Tse, D. D. Klug, and R. Ahuja, Phys. Rev. B 70, 214107 (2004).

[81] H. J. Xiang, Z. Li, J. Yang, J. G. Hou, and Q. Zhu, Phys. Rev. B 70, 212504 (2004).

[82] H. J. Choi, D. Roundy, H. Sun, M. L. Cohen, and S. G. Louie, Phys. Rev. B 66, 020513(R) (2002).

[83] Z. Zhao, S. Zhang, T. Yu, H. Xu, A. Bergara, and G. Yang, Phys. Rev. Lett. 122, 097002 (2019).

[84] A. P. Drozdov, M. I. Eremets, I. A. Troyan, V. Ksenofontov, and S. I. Shylin, Nature (London) 525, 73 (2015).

[85] X. Zhong, H. Wang, J. Zhang, H. Liu, S. Zhang, H.-F. Song, G. Yang, L. Zhang, and Y. Ma, Phys. Rev. Lett. 116, 057002 (2016).

[86] M. Iuga, G. Steinle-Neumann, and J. Meinhardt, Eur. Phys. J. B 58, 127 (2007).

[87] X.-Q. Chen, H. Niu, D. Li, and Y. Li, Intermetallics 19, 1275 (2011).

[88] Z. J. Wu, E. J. Zhao, H. P. Xiang, X. F. Hao, X. J. Liu, and J. Meng, Phys. Rev. B 76, 054115 (2007).

[89] Q. Li, H. Liu, D. Zhou, W. Zheng, Z. Wu, and Y. Ma, Phys. Chem. Chem. Phys. 14, 13081 (2012).

[90] V. L. Solozhenko, O. O. Kurakevych, and A. R. Oganov, J. Superhard. Mater. 30, 428 (2008). 